\title{
Aproximación a los modelos de género como herramienta para la intervención psicosocial en violencia de género
}

\author{
Trabajo de Reflexión y Planteamientos Metodológicos \\ Chiara Santoro ${ }^{6}$, María del Carmen Monreal-Gimeno \\ Gonzalo Musitu-Ochoa ${ }^{8}$, Belén Martínez-Ferrer ${ }^{9}$
}

\section{Introducción}

Las referencias a la violencia de género se tratan desde dos enfoques complementarios: por un lado, este problema social se identifica como la expresión máxima del patriarcado y del machismo y, por otro, como un hecho social normalizado, al que se le atribuye una escasa importancia, ya que se establece en un orden natural de las relaciones humanas, que se atribuye a predisposiciones naturales o atribuciones internas, $y$, por tanto, inmodificables ( $p$ ej, el agresor es un enfermo). Ambos aspectos coinciden en que se centran, principalmente, en la punta del iceberg de la violencia de género; es decir, la violencia física que, en determinados casos,

${ }^{6}$ Doctora en Ciencias Sociales, Universidad Pablo Olavide de Sevilla (España), y Dottorato di ricerca in Scienze della formazione e Psicologia, Universitá degli studi di Firenze (Italia). Investigadora asociada en Universidad Pablo Olavide, Sevilla, España. Correo: chiara.santoro@ hotmail.it

7 Doctora en Educación por la UNED. Profesora Titular, Departamento de Educación y Psicología Social, Universidad Pablo Olavide, Sevilla, España. Correo: mcmongim@upo.es

8 Doctor en Psicología, Universidad de Valencia. Catedrático Emérito de Psicología Social de la Familia, Departamento de Educación y Psicología Social, Universidad Pablo Olavide, Sevilla, España. Correo: gmusoch@ upo.es

9 Doctora en Psicología Social, Universidad de Valencia, España. Profesora Contratada Doctora, Departamento de Educación y Psicología Social, Universidad Pablo Olavide, Sevilla, España. Correo: bmarfer2@upo.es 
ocasiona la muerte de la mujer en manos del hombre. Si bien no se pueden negar estas formas extremas de violencia, resulta evidente que este análisis superficial limita las herramientas de comprensión de este problema y, sobre todo, impide desarrollar planes integrales y efectivos de intervención psicosocial. Para superar estas limitaciones es necesario analizar las creencias sobre las que se sustentan la amplia expresión de comportamientos, prácticas y creencias que construyen nuestra identidad y las formas de entender las relaciones interpersonales y, por tanto, en el que se fundamentan nuestras relaciones de género.

Además, el análisis de los modelos de género contribuye a superar la conceptualización de la violencia de género como un hecho de carácter excepcional al analizar la complejidad de las relaciones inter-género en el contexto social en el que se sustentan estas relaciones. De hecho, la violencia de género, lejos de ser un problema limitado al ámbito privado de una relación de pareja, es un proceso que se asienta en los procesos de definición de nuestra identidad, en los distintos contextos de socialización que construyen los contenidos de género y de las interacciones sociales y en el que diferencia entre mujer y hombre (Yubero Jimenez y Navarro Olivas, 2010; Monreal Gimeno, 2008; Monreal y Martínez, 2010). La definición excluyente de mujer versus hombre y viceversa, conlleva unos modelos normativos y excluyentes de masculinidad y feminidad que constituyen los elementos últimos del sistema hetoronormativo y patriarcal.

Como resulta evidente por los estudios antropológicos de Margaret Mead (1949) y David D. Gilmore (1990), estos contenidos de género se encuentran de forma transversal en todas las culturas. El análisis de estos contenidos y prácticas en la interacción social permite identificar los elementos de estabilidad de un sistema que sigue reproduciéndose a partir de una base binaria, jerárquica, diferencial y complementaria. Este análisis de la masculinidad y la feminidad cambia la concepción del género como una realidad estática. Comprender el género como "un mapa por el que transitamos" (Blanco, 2003), significa explorar las coordenadas donde nos situamos y ubicamos a los/las demás, y profundizar en las tensiones y diálogos generados en este tránsito. Desde este enfoque, la violencia de género es la expresión más evidente de un sistema de género configurado sobre bases 
duales y patriarcales, determinado por elementos contextuales y por la dinámica de cambio y resistencia de los modelos normativos de masculinidad y feminidad. En consecuencia, el estudio de estas dimensiones resulta fundamental para la intervención en la violencia de género.

\section{El género como concepto multidimensional y relacional}

El género se define como "la construcción cultural de la diferencia sexual, que da cuenta de un sistema primario de relaciones de poder y dominación, transhistóricas y transculturales" (Serret, 2011, p.73). Como señala Lamas (2016), el concepto de género resulta de gran relevancia en la definición del self, puesto que funciona como un 'filtro' cultural a través del cual se interpreta el mundo. Desde esta perspectiva, el género se construye en relación con tres dimensiones: la dimensión identitaria/personal, la dimensión cultural/histórica/social y la dimensión de poder. A su vez, la dimensión de poder género se articula en función de dos características:

- El binarismo masculino/femenino, que determina la existencia de dos géneros y su valoración diferencial, jerárquica, en un esquema cerrado, que no es dialéctico ni abierto. Lo masculino y lo femenino se definen como dos polos distintos, inalcanzables pero complementarios, fundamentado en el modelo de la diferencia sexual, donde lo masculino se individualiza como el polo positivo.

- La heterosexualidad normativa, que establece una modalidad de relación entre los sexos que se relaciona con el binarismo hombremujer y condena otras orientaciones sexuales. Según Lamas (1995), "Hoy la sexualidad se establece como un núcleo psíquico que define la identidad de la persona" (p. 72). En el régimen moderno que Foucault (1987) sitúa a comienzos del siglo XVIII, el sexo se establece como identidad, bajo los presupuestos de una "reproducción disciplinaria de la vida".

En este recorrido de diferenciación entre las distintas dimensiones del concepto de género, es importante enfatizar, retomando las palabras de Cedillo Hernández (2011), "el carácter relacional de las identidades y la importancia de los ordenamientos de género para que estas se constituyan” 
(p. 101). Las transformaciones vinculadas con las identidades genéricas o sexuales acontecen en los distintos ámbitos relevantes de la vida en interacción continua. Esta conceptualización dinámica del género nos permite comprender la dimensión transformadora del sujeto: "el género, por tanto, sería una forma de "Estar" en el mundo y no de "Ser", y esta visión nos ayuda a desencializar la experiencia" (Esteban, 2009, p. 34).

\section{Modelos de género}

El concepto de modelos de género o gendermodels aparece en las teorías de las diferencias de género o de la diferencia sexual, aunque a menudo carezcan de una definición conceptual y se suela referir a ellos como identidad de género o identidad sexo-genérica, resultado de la construcción socio-cultural de feminidad y masculinidad a nivel de su integración identitaria. Como señala Connell (1997), el género es un sistema que permite ordenar y comprender la práctica social que, en el contexto actual está definida por el escenario reproductivo. Así, los modelos de género responden a determinadas necesidades contextuales de organización social. De la misma forma, Monreal y Martínez (2010) subrayan el carácter social de los modelos de género, categorizados bajo la forma de esquemas cognitivos. Estos esquemas de género nos indican como son o como deben ser los hombres y las mujeres, sencillamente por el hecho de adscribirse a una categoría (masculina o femenina). Por consiguiente, la modelación de género es un proceso constitutivo del orden social, que se integra en el individuo como una dimensión cognitiva, con una clara función adaptativa, ya que facilita la adquisición de información desde el entorno. En consecuencia, los modelos de género, más allá de ser únicamente estructuras cognitivas que estructuran las creencias y conductas, constituyen un conjunto de prácticas incardinadas en el sistema de relaciones de género.

Desde el modelo patriarcal dominante, la masculinidad y la feminidad estructuran una realidad binaria en la que 'masculino' y 'femenino' son extremos opuestos, excluyentes y a la vez complementarios (García y Freire, 2003). En otros términos, "la mujer y lo femenino representan el límite, la frontera de la masculinidad, lo abyecto" (Lomas, 2004, p. 52). Si se asume la superioridad física, intelectual, social y hasta espiritual de lo masculino sobre lo femenino, se termina justificando un sistema de opresión, domi- 
nación y discriminación sobre las mujeres y sobre todos aquellos hombres que se distancian de aquel modelo (Rocha Sánchez y Lozano Verdugo, 2014). La heterosexualidad normativa, fundamentada sobre el mito de la complementariedad entre los modelos, sustenta tal sistema de poder. La complementariedad prima sobre la igualdad justamente por su conexión con el mantenimiento de un determinado orden social, que sin comprender la diferencia podría romperse, con consecuencias que se perciben más graves, ya que alcanzan una dimensión colectiva (Mateos Sillero, 2013).

\section{Modelo de masculinidad}

El hombre no nace hombre, deviene en el poder que se ejerce, constituyendo así el "babitus masculino" (Mateos Silleros, 2013; Rodríguez Menéndez, 2003). La masculinidad hegemónica es un modelo ideal, y por tanto inalcanzable. La marca natural, inscrita en los cuerpos masculinos, tiene que ser continuamente demostrada, su potencialidad tiene que ser afirmada a nivel social, tiene que hacerse efectiva. Para Guash (2000), la masculinidad se define por tres pilares, que reflejan tres oposiciones fundamentales que representan los límites del "ser masculino": (1) el hombre no es femenino y, por tanto, se rechaza el universo femenino y de las mujeres; (2) el hombre no es un niño y, en consecuencia, se prioriza la autonomía personal; y (3) el hombre no ama a otros hombres. Por tanto, la homofobia se expresa en un fuerte individualismo y en la falta de compromiso, que se conecta con la insolidaridad y desigualdad en las relaciones con las mujeres (Valcuende y Blanco, 2003).

En general, un hombre que sigue el modelo de masculinidad dominante debe ser autónomo, activo, inteligente, superior, racional, con control emocional, hipersexual, heterosexual, con rol de proveedor, que toma las decisiones y dominante (Lomas, 2004; Rocha Sánchez y Lozano Verduzco, 2014). El ámbito de desarrollo fundamental se asocia a la esfera pública; es allí donde la masculinidad puede demostrarse en su habitus viril, dominante y en su estilo impersonal. En el trabajo se define el periodo adulto de la hombría, y se identifican las contradicciones existentes entre el ideal del varón libre indomesticable y el sentido de responsabilidad respecto a "los más débiles", su familia, las mujeres, los niños/as (Fuller, 1997). La masculinidad es un arma de doble filo; si bien otorga reconocimiento social 
y prestigio, poder y privilegios, es una larga carrera hacia un objetivo inalcanzable que implica muy a menudo dolor físico y psíquico, incomodidad y molestia, tensión y conflicto, así como un combate sin fin a menudo dirigido hacia uno mismo (Matud, 2008; Olavarría, 2001; Valdés y Olavarría, 1998).

\section{Modelo de feminidad}

El orden simbólico comprende la feminidad como el otro pilar que complementa y delimita la masculinidad. La imposibilidad de definición que reside en la masculinidad se resuelve en la feminidad. La complementariedad del modelo y su consecuente heteronormatividad vincula la masculinidad a la feminidad, aunque se mantenga la jerarquía de la masculinidad y el miedo a la feminidad, como posible pérdida de virilidad, elementos que perpetúan estructuras de dominación y de minusvaloración de la feminidad. Si por un lado la mujer se asocia a la virginidad, al culto de la Virgen María, en su pasividad o ausencia de sexualidad, por otro lado, la vinculación de la mujer con el cuerpo, la identifican con la insaciabilidad de su deseo sexual, que alejaría y tentaría el hombre, y sería peligroso para la consecución de sus objetivos. (Santos Velásquez, 2009).

La disposición sumisa conlleva consecuencias respecto a las actitudes y a las conductas asociadas a la feminidad: la abnegación, la resignación, el silencio, la abstención, la abstinencia, la docilidad y la entrega suelen ser consideradas características femeninas. Además, la feminidad implica la inhibición de la agresividad, que, si se manifesta, genera sentimientos de vergüenza y culpabilidad. Esta permanencia en la falta del poder genera también una escasa capacidad de reconocimiento individual de las propias competencias y capacidades, debido a un sentimiento general de inferioridad (Rodríguez Menéndez, 2003).

A través de la maternidad, la feminidad vincula la existencia de las mujeres al ser para otros, generando un habitus codependiente, que asocia la subjetividad de las mujeres al reconocimiento y al amor de los otros/ de las otras. Esta subordinación, esta renuncia a sí misma como sujeto, la vinculación de la propia identidad a la existencia y al cuidado del otro, se configura como un acto de amor. A través del amor y de la virtud las 
mujeres consienten un sistema de subordinación social que las controla y las domina, puesto que amando a sus seres queridos más que a sí misma, la mujer se convierte en un ser virtuoso, que finalmente podrá ser valorado y reconocido socialmente en su autoexclusión del poder en la medida en que el poder de la mujer se circunscribe a la esfera privada (Mateos Sillero, 2013). Los roles de género también se basan en el eje de complementariedad y subordinación al hombre (De Barbieri, 1993). El amor romántico, el mito de la media naranja, otorga sentido a esta división desigual en términos de prestigio, de autonomía económica, de poder.

En consecuencia, el ámbito de desarrollo de la feminidad es el ámbito privado, donde se desarrollan las tareas de cuidado y las relaciones de amor, el trabajo reproductivo: este incluye la atención no solo a las necesidades de carácter material de la familia, sino la atención psíquica, intelectual, emocional, afectiva, sensual y sexual. Como afirma Gil Calvo (2000), las mujeres "no son libres de expresarse a voluntad, pues siempre deben tener en cuenta su familia, de la que dependen en mayor o menor medida" ( $\mathrm{p}$. 98). El amor termina produciendo relaciones de dependencia. El desarrollo profesional y personal pasa a segundo plano, y no asume un papel central y prioritario como en la masculinidad. A partir de esta priorización, y del lugar tradicional de las mujeres como tejedoras de las relaciones humanas, se asocia a la feminidad el habitus expresivo, en oposición con el habitus impersonal de la masculinidad. El habitus expresivo reconoce la existencia del otro y lo pone en el centro, en detrimento de sí mismo.

\section{Violencia hacia la mujer desde la perspectiva de los modelos de género}

Dentro de los modelos de género, las creencias y estereotipos de género orientan y guían las relaciones entre ambos géneros. Hemos podido apreciar como de acuerdo con las creencias de género, las mujeres se consideran menos valiosas que los hombres y subordinadas a los mismos. Hasta el punto de que en este servir y atender los deseos de los hombres puede considerarse que por su inferioridad pueden ser controladas por ellos y hacer uso de la violencia para someterlas. En consecuencia, las "agresiones sexuales" que podríamos considerar que están en la base de la violencia de género y la explican, no solo se conceptualizaron como como pérdida de la honra femenina y, por ende, familiar que debía ser resuelto entre varones, 
sino sobre todo como un acto de violencia ejercida hacia una o más mujeres (Monreal, 2002).

La violencia, y en particular la agresión sexual, se interpreta como una medida de coacción para el conjunto de las mujeres con el objeto de que estas asuman el conjunto de normas y de prácticas que definen a "las buenas mujeres" y a "las malas mujeres". Como señala Monreal (2009) las buenas mujeres pertenecen a un solo hombre, preferiblemente a través del matrimonio, no salen solas a ciertas horas, no adoptan actitudes provocativas o insinuantes. De hecho, existe un creciente volumen de investigación en la que se concluye que la motivación de las agresiones sexuales no es el sexo o el deseo sexual, sino la agresión y la dominación. Desde los posicinamientos feministas, se entiende la agresión sexual como una forma de agresión y de hostilidad hacia la mujer entendida en sentido genérico; es decir, hacia todas las mujeres. En este sentido, la violencia es un vehículo para el ejercicio del poder, para el control de las mujeres. La agresión ya sea real o su amenaza, funciona además, como un metalenguaje nada sutil por el que ha señalado a la mujer el rol en el que se debe situar; es decir, una posición de inferioridad, relegada al espacio privado, ajena al mundo masculino. De hecho, cuando las mujeres entran masivamente en el ámbito público, el acoso sexual constituye un elemento de persuasión para indicarle cuál es su sitio

\section{Factores estructurales de la violencia de género}

La agresión sexual o la violación ha sido tradicionalmente comprendida en el marco de los mitos que la conciben como un producto inevitable de las necesidades sexuales masculinas. En consecuencia, como afirma Monreal (2002), el modelo de "masculinidad" y orientación heterosexual se encuentran imbricadas en un contexto de dominación económico, social, político, ideológico, que justifica no solo la agresión sexual, sino también la violencia hacia las mujeres. De esta manera, la "violencia" se asocia con la "masculinidad", de modo que la violación ha sido asimilada a una actividad sexual ligada a la "hombría" y no primordialmente como una actividad violenta. Además, esta asociación entre masculinidad y heterosexualidad dota al acto violento, y especialmente a la agresión sexual, de conductas con una elevada carga simbólica. En este sentido, a través de estas agresiones 
el hombre debe probar su masculinidad. Por el contrario, la identidad femenina ha sido construida históricamente como carente de una sexualidad propia, surgiendo el binomio del hombre activo/mujer pasiva, el hombre que conquista y la mujer que se resiste (como corresponde a su papel) pero que en realidad quiere ser conquistada: "cuando dicen no, quieren decir sí".

La violencia contra las mujeres solo puede ser entendida dentro de una concepción de la mujer como una propiedad masculina, que puede usar el varón según le apetezca. Por ello, la violencia contra las mujeres refleja la cosificación de la mujer; es decir, la mujer es un objeto al servicio del hombre (Monreal, Povedano y Martínez-Ferrer, 2014). Este modelo androcéntrico de relación entre hombres y mujeres que se expresa en un tipo de sexualidad es el que ha dificultado la visibilización de prácticas como la violación dentro del matrimonio o el abuso sexual realizado por hombres cercanos a la víctima y las prácticas violentas. Para Monreal (2009), este modelo: "comporta unos determinados rasgos: coitocentrista, prima la cantidad frente a la calidad de los encuentros sexuales, deseo sexual masculino incontrolable, negando la posibilidad de una sexualidad femenina autónoma, guiada por su propia iniciativa" (p.183). En el marco de este modelo, la violencia se justifica en aras de un modelo sexual, invisibilizando que, como se señala desde la perspectiva feminista, la violación implica, especialmente, el uso de la fuerza, la coerción y la imposición, cosificando a la mujer y evidenciando hostilidad y desprecio frente al género femenino. Por ello, la violencia evidencia un modelo de relación entre hombres y mujeres de dominación que se construye en el proceso de socialización (Monreal y Martínez, 2010).

Como señala Monreal (2009), aquellos aspectos de los estereotipos que subyacen a las agresiones sexuales y que las justifican se pueden agrupar en tres dimensiones:

- La violación como producto inevitable de las necesidades sexuales masculinas; es decir, resultado de un impulso irrefrenable.

- La violación como un riesgo inherente asociado a la categoría de ser mujer; la violación es algo que se puede hacer a la mujer por el mero hecho de serlo. 
- La violación como una consecuencia de un modelo en el que se justifica en los varones una cierta dosis de agresividad, como si parte inherente de la masculinidad fuera una cierta dosis de violencia.

En síntesis, los estereotipos se articulan en torno a la idea de superioridad del varón en todos los órdenes y, en especial, en la interacción con las mujeres en todos los ámbitos. De este modo, se evidencia que la agresión implica el ejercicio del poder porque, para dominar a veces hay que intimidar y coaccionar.

Por lo tanto, los vínculos entre las agresiones contra las mujeres y las pautas de conductas aprobadas socialmente, en cuanto a las normas derivadas de los estereotipos de género nos ayudan a comprender mejor, la falta de sensibilidad y la normalización de un sector amplio de la sociedad hacia la violencia contra las mujeres. Estos patrones son el resultado de un proceso socio-histórico y se perpetúan por medio de su transmisión en la socialización, fundamentada en la desigualdad entre los sexos. Estos estereotipos justufucan la violencia y culpabilizan a las mujeres en estas situaciones de violencia y acoso (Monreal, 2009).

\section{Claves para la intervención psicosocial en la violencia de género desde los modelos de género}

En este breve análisis, se ha evidenciado como, a partir de nuestro posicionamiento teórico respecto al concepto de genero, los modelos de feminidad y de masculinidad se configuran como elementos claves en la comprensión de la situación de desigualdad. Es evidente que, por lo menos en el contexto europeo, en los últimos 60 años, gracias a los movimientos feministas, y a los movimientos LGBTQI, se ha producido una quiebra en muchos de los ejes centrales de los modelos de género: la entrada en el espacio público de las mujeres, la crisis del modelo de maternidad normativa, la emergencia de nuevos modelos de masculinidad a partir de los movimientos homosexuales, que se oponían a la normatividad del modelo justo a partir de su fundamento, la heterosexualidad, revindicando un ser varón no condicionado por determinadas prácticas sexuales (Santoro, 2014).

Estas fisuras en el modelo hegemónico no han conllevado el fin de los modelos de género sobre los que se construye una estructura de género bi- 
naria, pero han insertando otras realidades creando múltiples feminidades y masculinidades (Santoro y Monreal, 2016). Además, la profundización en los estudios de género, y la puesta en crisis de las mismas nociones binaria de masculinidades y de feminidades por parte de los movimientos transgénero y queer abre nuevas interpretaciones en la misma teoría de modelos y categorías socialmente definidas.

Este ensanchamiento de los límites normativos de los modelos se evidencia en una contradicción entre las prácticas y las creencias relativas a los modelos de género. Si bien la estructura socio económica ha necesitado involucrar a las mujeres en el ámbito laboral, este hecho produce inevitablemente una serie de reajustes con respecto a la labor de cuidado de los hombres. Así, en el ámbito educativo, que se caracteriza por su constante feminización y, en consecuencia, un constante aumento de las posibilidades de relaciones socio-afectivas, la estructura binaria y los modelos normativos de masculinidad y feminidad siguen siendo el material de género que constituye y regula la sociedad en la que vivimos.

De hecho, una de las implicaciones para la intervención desde el ámbito educativo alude no tanto a evidenciar el nivel de persistencia de los modelos de género normativos, como, y sobre todo, a analizar el nivel de contradicción existente entre las experiencias personales de cada uno y cada una y la consciencia y la presión ejercida por el estereotipo. En este sentido, cabe destacar que, una vez que se han elaborado los esquemas, las características forman un entramado con cierta autonomía, de manera que las modificaciones en las características del grupo original no afectan al entramado de información asociada a ese grupo (Monreal y Martínez, 2010), por lo que es necesario tener en cuenta los modelos, esquemas y estereotipos de género.

Además, existe un desfase creciente entre el modelo de masculinidad normativa y el de feminidad. El modelo de feminidad ha sido objeto de una profunda revisión que ha conllevado importantes cambios en las prácticas y en la vida de las mujeres. Sin embargo, el modelo de masculinidad permanece estable, posiblemente porque los hombres apenas se han involucrado como sujetos activos en las luchas para la igualdad. De hecho, la participación de los hombres en la lucha por la igualdad es una línea emergente de 
intervención que, de momento, se visibiliza en la creación, aun minoritaria, de grupos y colectivos de hombres para la igualdad o feministas. En este sentido, los movimientos asociados con la nueva masculinidad, se han asociado a una pérdida de poder" (Sánchez-Palencia, 2001). La visibilización de los hombres en un marco ajeno al modelo tradicional de masculinidad y su reconocimiento como un grupo social específico, caracterizado por su pertenencia a un sexo y a un género determinado, ha devenido un proceso de gran relevancia para cuestionar el modelo de dominación imporante, lo que, a su vez, conlleva sus dificultades (Blanco, 2003), pero es, indudablemente, un desafio en las nuevas políticas y acciones de intervención, en la medida en que, como venimos subrayando, los modelos de género son dinámicos y surgen desde y en la interacción social.

El análisis de los modelos de género, asumiendo éstos como conceptos relacionales que se constriuyen en y desde la interacción proporciona una mayor comprensión de la persistencia de la violencia de género, sobre todo en jóvenes, y en aquellas situaciones en que "sus mujeres" ejercen su autonomía y por tanto escapan a su control (se han separado, han iniciado una nueva vida etc.). La violencia de género, por tanto, se identifica con la expresión de un proceso estructurado a partir de un núcleo de poder desigual, complementariedad y exclusividad en las relaciones de género, que se alimenta además de mitos y otras creencias del amor romántico. La resistencia de ideas ancladas a una visión tradicional de la masculinidad y de la feminidad, más allá de los cambios casi inevitables inducidos por las practicas, que como hemos visto están claramente vinculados con el ingreso de las mujeres en la educación y en el mercado del trabajo, puede causar diferentes reacciones, que conllevarían relaciones desiguales y reivindicaciones de poder y de afirmación.

La crisis de la masculinidad, tema muy actual en los estudios de género, podría vincularse con la persistencia y el recrudecimiento de la violencia de género sobre todo entre los jóvenes. Como hemos visto, la mayor resistencia de modelos fijos en los hombres, tanto asociados a la masculinidad como a la feminidad, podría provocar una mayor reacción a los cambios percibidos en las practicas, que determinarían fuertes contradicciones y consecuentes frustraciones, entre los mandatos, los roles, las expectativas respecto a la 
propia vida y a las relaciones que de estas se producen, y las efectivas posibilidades de afirmarlas en la cotidianeidad y en sus relaciones reales.

No obstante, es necesario rescatar que los modelos de género, más allá de ser fijos, tienen un potencial adaptativo. Como afirman Monreal y Martínez (2010), los esquemas de género son estructuras dinámicas de conocimiento que cambian en función del contexto social en el que se desarrollan. A la vez, como ya hemos subrayado, los modelos nos sirven como estructura de referencia tanto para la construcción de nuestra identidad de género como para nuestras interrelaciones con el mundo exterior. Por estas razones, resulta fundamental encontrar nuevos apoyos, "ya que anclajes anteriores en valores y estilos de vida tradicionales, en el caso de las mujeres, y la masculinidad hegemónica, en el de los hombres, no sustentan el desarrollo de características innovadoras" (Del Valle et al., 2002, p. 44).

A través de nuestra acción individual, y sobre todo con nuestra intencionalidad, aunque con ciertas resistencias, no solo podemos contribuir a un cambio en los modelos de género normativos, sino también generar un cambio en las relaciones, que amplia la estructura binaria que limita las posibilidades de pensamiento, de acción, de comprensión de las sociedades en las que vivimos. Por ello, el estudio de los modelos de género, que tenga en cuenta no solo las resistencias sino también los elementos emergentes de cambio, puede permitir una intervención enfocada en la situación real de igualdad y los factores estructurales que están en la base de la violencia de género, orientados a su prevención real y no exclusivamente en su contención o en la atención a las "víctimas". 


\section{Referencias bibliográficas}

Badinter, E. (1994). XY, la identidad masculina. Bogotá: Norma.

Blanco, J. (2003). Las expectativas sobre los varones. En Valcuende Del Río, y J. M., Blanco López, J., Hombres. La construcción cultural de las masculinidades. (pp. 212-225) Madrid: Talasa Ediciones.

Cedillo Hernández, P. (2011). Los avatares del cuerpo en la constitución de la identidad: Un acercamiento a través de la obra de Pierre Bourdieu y Marcel Mauss. En GénEros, (9), 99-120.

Connell, R.W. (1997). La organización social de la masculinidad. En Valdés, T.,y Olavarría, J. (Eds.) Masculinidades, Poder y crisis (pp. 31-48). Santiago: ISIS international/ Flacso Chile.

De Barbieri, T. (1993). Sobre la categoría género. Una introducción teóricometodológica. Debates en Sociología, (18), 145-169.

Del Valle, T., Apaolaza, J.M., Arbe, F., Cucó, J., Díez, C., Esteban, M.L., Etxebarria, F., y Maquieira, V. (2002). Modelos emergentes en los sistemas y las relaciones de género. Madrid: Narcea.

Esteban, M. L. (2009). Identidades de género, feminismo, sexualidad y amor: los cuerpos como agentes. Politica y Sociedad, 46 (1y2), 27-41.

Foucault, M. (1987) La historia de la sexualidad I. La voluntad de saber. Madrid.

Fuller, N. (1997). Identidades masculinas. Varones de clase media en el Perú. Lima: Pontificia Universidad Católica del Perú.

García, A., y Freire, M. (2003). Desarrollo del género en la feminidad y en la masculinidad. Madrid: Narcea.

Gilmore, D. (1990). Manhood in the Making: Cultural Concepts of Masculinity. New Haven, Conn.: Yale University Press.

Guasch O. (2000). La crisis de la Heterosexualidad. Barcelona: Ed. Laertes.

Lagarde, M. (2005). Para mis socias de la vida. Madrid: Horas y Horas. 
Lamas, M. (2016). Género. En Moreno, O., y Alcántara, E. (Eds.) Conceptos clave en los estudios de género (pp.155-170). Ciudad de México, Universidad Nacional Autónoma de México.

Lamas, M. (Ed.). (1995). El género, la construcción cultural de la diferencia sexual. México: Porrúa.

Lomas, C. (2004). Los chicos también lloran: identidades masculinas, igualdad entre los sexos y coeducación. Barcelona: Paidós.

Marqués, J. V., y Osborne, R. (1991). Sexualidad y sexismo/Josep-Vicent Marqués, Raquel Osborne. Fundación Universidad-Empresa.

Mateos Sillero, S. (2013). Construcción de la feminidad normativa y sujeto político. Investigaciones feministas, 4, 297-321.

Matud Aznar, M.P. (2008). Género y Salud. Suma Psicológica, 15(1), 75-94.

Mead, M. (1994- ed. or. 1949). Masculino y femenino. Madrid: Minerva.

Monreal-Gimeno, M.C. (2008). Esquemas de género y violencia hacia la mujer. En A.M. Tagle, y R Valpuesta,. (Eds.) Ni el aire que respiras. Pensamiento cientifico ante la violencia de Género (pp. 89-107). Sevilla: Colección Señales. Fundación Obra Social Cajasol.

Monreal, M.C., y Martínez, B. (2010). Esquemas de género y desigualdades sociales. En L. Amador, L. y M. C Monreal (Eds.), Intervención social y género (pp. 73-94). Madrid: Narcea.

Monreal-Gimeno, M. C., MArtinez-Ferrer, B., y Povedano, A. (2014). Modelo ecológico de los factores asociados a la violencia de género en parejas adolescentes. Journal for Educators, Teachers and Trainers, 5 (3), 105-114.

Olavarría, J. (2001). ¿Hombres a la deriva? Poder, trabajo y sexo. Santiago: Flacso Chile.

Rocha Sánchez, T., y Lozano Verduzco, I., (eds.), (2014). Debates y reflexiones en torno a las masculinidades: Analizando los caminos hacia la igualdad de género. México: UNAM. 
Rodríguez-Menéndez, M. C. (2003). La configuración del género en los procesos de socializacion. Oviedo: KRK Ediciones.

Sánchez Palencia, C., y Hidalgo, J. (Eds.) (2001). Masculino plural: construcciones de la masculinidad. Lleida: Universidad de Lleida.

Santoro, C. (2016). Los modelos de género desde una perspectiva intercultural: un proyecto de investigación entre Italia, España y Argentina. $V$ Congreso Interoceánico de Estudios Latinoamericanos. UNCUYO, Mendoza, Argentina.

Santoro, C., y Monreal Gimeno, M. C. (2016). Los modelos de género desde una perspectiva intercultural: un proyecto de investigación para el desarrollo de políticas de género en el ámbito universitario en Italia, España y Argentina. Cuestiones pedagógicas, (25), 65-76.

Santos Velásquez, L. (2009). Masculino y femenino en la intersección entre el psicoanálisis y los estudios de género. Bogotá: Universidad Nacional de Colombia.

Serret, E. (2011). Hacia una redefinición de las identidades de género. GénEros, época 2, año 18 (9), 71-97.

Valcuende, J. y Blanco, J. (Eds.) (2003). Hombres. La construcción cultural de las masculinidades. Madrid: Talasa.

Valdés, T., y Olavarría, J. (Eds.) (1998). Masculinidades y Equidad de género en América Latina. Santiago: FLACSO Chile.

Yubero Jímenez, S., y Navarro Olivas, R. (2010) Socialización de género. En Amador, L. y Monreal, M.C. (Eds.) Intervención social y género (pp. 43-72). Madrid: Narcea. 